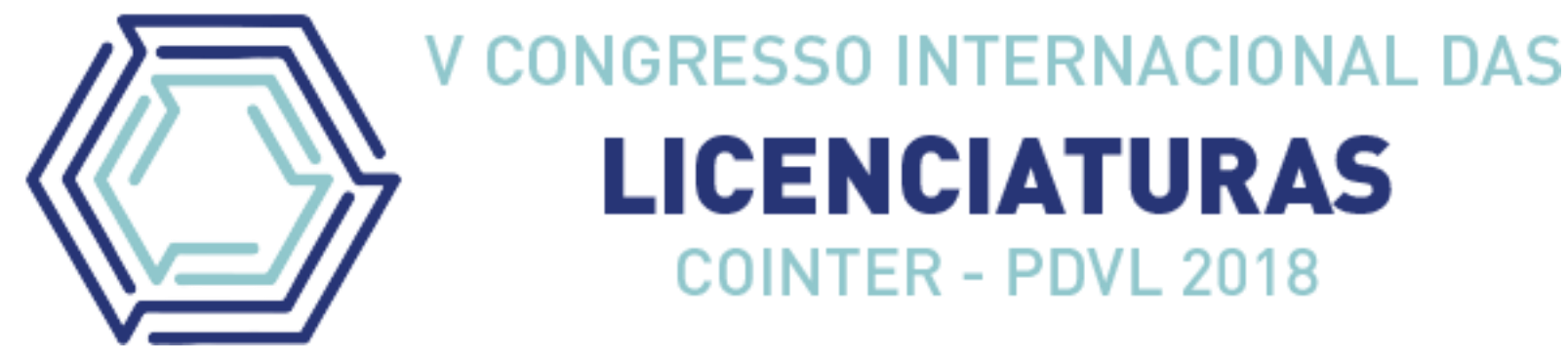

\title{
RESOLUÇÃO DE PROBLEMAS (RP): UMA PROPOSTA DIDÁTICA DIFERENCIADA PARA O ENSINO DE QUÍMICA
}

\section{PROBLEM SOLVING (PR): A DIFFERENTIATED TEACHING PROPOSAL FOR CHEMISTRY EDUCATION}

\author{
Apresentação: Comunicação Oral \\ Jerônimo Ferreira Mendes ${ }^{1}$; Kymberli Francisca de Souza²; Thainá Maria da Silva Carvalho ${ }^{3}$ \\ Fernanda Karoline Silva ${ }^{4}$; Sanderson Hudson da Silva Malta ${ }^{5}$
}

\section{DOI: https://doi.org/10.31692/2358-9728.VCOINTERPDVL.2018.00144}

\section{Resumo}

No cenário educacional em que se encontra os discentes, pode-se perceber a existência de dificuldades por parte dos estudantes no processo de ensino-aprendizagem na área da química. Visto que, existem inúmeros motivos que levam ao mesmo a essa situação. É necessário encontrar os motivos que levam a essas circunstancias. Diante desse contexto a proposta é aplicar a Resolução de Problemas (RP) baseada nas provas de anos anteriores do ENEM (Exame Nacional do Ensino Médio) para minimizar as dificuldades dos alunos nos conteúdos, pois RP desenvolve o caráter motivador nos estudantes além de outros pressupostos, que possibilita desenvolvimento de competências que colaborem com um maior rendimento nos processos seletivos para ingresso no ensino superior. $O$ presente artigo se assegura nas ações metodológicas do Ciclo da Experiência Kellyana (CEK) atrelado a ferramenta didática RP realizados nas escolas estaduais circunvizinhas ao IFPE Campus Vitória de Santo Antão, parceiras do Programa Internacional Despertando Vocações para Licenciaturas, na qual as ações e atribuições estão inseridas. Esse ciclo é constituído por cinco etapas que se conectam: (i) Antecipação; (ii) Investimento; (iii) Encontro; (iv) Confirmação ou Desconfirmação; e (v) Revisão Construtiva. Nesse sentido os resultados revelam que o maior impasse que contribui negativamente para aprendizagem dos alunos é a falta de domínio em cálculos, sendo que por esta razão eles alegam que o conteúdo que apresenta mais complicação são os Cálculos Estequiométrico, além disso a falta de afetividade com a disciplina também se mostra presente. Nesta perspectiva, prevê-se que deve ter uma iniciativa para diminuir esse efeito, sendo que a maior demanda recair na deficiência de cálculos, além disso deve considerar que há necessidade do incentivo, em levantar a estima dos estudantes para criarem a percepção que Química não é algo tão difícil ou impossível de se aprender. Sendo assim, a RP ganha espaço nesse cenário.

Palavras-Chave: Ensino-aprendizagem, Resolução de problemas, ENEM, Ciclo da Experiência Kellyana

\footnotetext{
${ }^{1}$ Licenciatura em Química, Instituto Federal de Pernambuco - IFPE, pietromendes0123@gmail.com

${ }^{2}$ Licenciatura em Química, Instituto Federal de Pernambuco - IFPE, kymberlisouza@hotmail.com

${ }^{3}$ Licenciatura em Química, Instituto Federal de Pernambuco - IFPE, thainamscarvalho@outlook.com.br

${ }^{4}$ Licenciatura em Química, Instituto Federal de Pernambuco - IFPE, fernandakds@hotmail.com

${ }^{5}$ Especialista, Instituto Federal de Pernambuco - IFPE, sanderson.malta@vitoria.ifpe.edu.br
} 


\begin{abstract}
In the educational context in which the students are, it is possible to perceive the existence of difficulties on the part of the students in the teaching-learning process in the area of chemistry. Since there are many reasons why this is the case. It is necessary to find the reasons that lead to these circumstances. In view of this context, the proposal is to apply Problem Solving (PR) based on previous years ENEM (National High School Examination) tests to minimize the difficulties of students in content, since RP develops the motivating character in the students besides other assumptions, which enables the development of competencies that collaborate with a higher income in the selective processes for admission to higher education. This article assures itself in the methodological actions of the Kellyana Experience Cycle (CEK) linked to the RP didactic tool carried out in the state schools surrounding the IFPE Campus Vitória de Santo Antão, partners of the International Program Awakening Vocations for Undergraduate Courses, in which the actions and attributions are inserted. This cycle consists of five stages that connect: (i) Anticipation; (ii) Investment; (iii) Meeting; (iv) Confirmation or Disconfirmation; and (v) Constructive Review. In this sense, the results reveal that the greatest impasse that contributes negatively to students' learning is the lack of mastery in calculations, being that for this reason they claim that the content that presents more complication are Stoichiometric Calculations, besides the lack of affectivity with discipline is also present. In this perspective, it is anticipated that it should have an initiative to reduce this effect, and that the greater demand is due to the deficiency of calculations, in addition it must consider that the incentive is necessary, in raising the esteem of the students to create the perception that Chemistry does not is something so difficult or impossible to learn. Thus, the RP gains space in this scenario.
\end{abstract}

Keywords: As mesmas palavras-chave traduzidas em inglês.

\title{
Introdução
}

De acordo com os Parâmetros Curriculares Nacionais de Química (PCN) "Química é uns dos componentes curriculares que contribui para a explicação da natureza e suas transformações, do próprio ser humano e suas ações, colaborando também para o desenvolvimento tecnológico." (BRASIL, 2006, p. 102). Sendo assim, nos últimos anos o ensino aprendizagem nessa área transformou-se em premente preocupação, visto que os alunos não estão alcançados de forma objetiva os conteúdos destinados a essa área, pois apresentam dificuldades em conceitos necessários para compreender ou colocar em prática esse ramo científico.

Em virtude disso, é notória a necessidade de uma solução para minimizar esse problema, pois o maior impasse que leva aos mesmo a essa situação é a carência nos domínios de cálculos. Nesta perspectiva, existem vários métodos didáticos diferentes que podem ser vistos para enfrentarmos essa dura realidade. Dentro dessa circunstância, emerge a necessidade de buscas alternativas que desperte o interesse do aluno, motivando assim o mesmo, a 
interpretar os fenômenos que estão ao seu alcance, para então assim desenvolver melhor o seu intelecto. Viana, (2014) considera a avaliação como principal processo para o ensino e aprendizagem, pois é ela quem irá promover as transformações a partir de seus resultados e dados construídos, dentro de tomadas de decisões compartilhada.

Devido à ausência das relações entre os conteúdos e cotidiano dos discentes a aprendizagem fica submersa, pois muitas vezes não desperta no mesmo o interesse de aprender e entender. Um modelo diferenciado, Resolução de Problemas vem sendo usado para esse contexto e é de importância fundamental, por permitir desenvolver não só a reprodução de conceitos, mas também conhecimento processual e competência que muitas vezes, os cidadãos têm de mobilizar quando enfrentam problemas no seu que cotidiano.

Além do mais, Resoluções de Problemas assume caráter cognitivo, em que proporciona aos alunos oportunidades para se interrogarem sobre os processos pelo qual aprendem, tornando-se conscientes deles, quer sobre as causas das suas dificuldades quando não aprendem. Logo pode-se perceber que a proposta oferece aos alunos não só a exigência de raciocínios e cálculos, mas também lhe proporcionam a percepção do motivo pela qual não aprende, ou seja, sua maior dificuldade.

Diante desse cenário, a pesquisa em si tem como caráter analisar de forma minuciosa os principais motivos que levam aos estudantes a terem um mal desempenho ou não, na cultura cientifica restrita a Química. E através do reconhecido aprimorar a resolução de problemas para minimizar os efeitos dessa problemática. Tendo como finalidade fortalecer um maior desempenho e afetividade com as questões do Exame Nacional do Ensino Médio (ENEM), e, por conseguinte com os processos seletivos.

\section{Fundamentação Teórica}

São muitos os fatores que irão resultar em dificuldades de aprendizagem pela grande maioria dos alunos e para amenizar esse problema, é necessário enfatizar as pesquisas nesta problemática, procurando compreender os diversos fatores que a cercam (SILVA, 2013).

A Química é uma disciplina relativamente jovem, sendo um componente curricular obrigatório. No Brasil, essa ciência foi inserida como disciplina regular a partir de 1931 (LIMA, 2013). Contudo, os alunos apresentam dificuldades no processo de aprendizagem nesse ramo cientifico, pela qual, desenvolve nos alunos a desmotivação e o desinteresse de aprender. A maioria dos alunos sente muita dificuldade em entender determinadas questões de Química, 
principalmente as que necessitam de cálculos matemáticos, pois muitas vezes essas questões exigem os conceitos químicos para serem resolvidas, ou seja, uma questão de cálculo depende da teoria para ser resolvida (SILVA, 2013). Além do mais. Na maioria das escolas tem-se dado maior ênfase à transmissão de conteúdos e à memorização de fatos, símbolos, nomes, fórmulas, deixando de lado a construção do conhecimento científico dos alunos e a desvinculação entre o conhecimento químico e o cotidiano. Essa prática tem influenciado negativamente na aprendizagem dos alunos, uma vez que não conseguem perceber a relação entre aquilo que estuda na sala de aula, a natureza e a sua própria vida (MIRANDA; COSTA, 2007). Falando sobre isto Paulo Freire reflete que a tarefa do professor é "a de ensinar e não transferir conhecimento" (FREIRE, 2008, p. 47).

A resolução de problemas é uma das estratégias pedagógicas que favorecem ao aluno um aprendizado devido ao estimulo pelos desafios (VYGOTSKY, 1998, p. 129-130 apud ZUCHI, 2004, p. 54). Num ambiente de aprendizagem em que o diálogo é fundamental, a relação professor/aluno não pode ser de autoritarismo de nenhum dos lados. O professor deve ocupar uma posição de mediador entre o conhecimento e os alunos e é preciso que ambos sejam os "coautores" do processo ensino-aprendizagem (GASPARIN, 2003, p. 2). Resolução de exercícios e resolução de problemas são metodologias diferentes. Enquanto na resolução de exercícios os estudantes dispõem de mecanismos que os levam de forma imediata, à solução, na resolução de problemas isso não ocorre, pois, muitas vezes, é preciso levantar hipóteses e testá-las (PARANÁ, 2006, p. 43)

A resolução de problemas contempla a concepção do conhecimento, visto que estimula e amplia a rede de significação dos elementos apreendidos na realidade. Estabelece uma relação de continuidade e ruptura na análise, levantamento dos dados e também na construção de hipóteses. Permite a reflexão e o pensamento crítico em todas as etapas da resolução (ANASTASIOU, 2012). Além disso, o processo de resolução de problemas potencializa a interação entre aluno-professor e aluno-aluno uma vez que ocorrerá uma permanente discussão dos conteúdos o que poderá propiciar uma construção coletiva do conhecimento (LACERDA et al., 2012). A literatura da área de Ensino de Ciências também explicita diferenças entre exercício e problema da vida real. Em problemas da vida real é necessário que haja inicialmente uma problematização do enunciado para que as questões centrais do problema possam emergir desse contexto de acordo com, Santos e Schnetzler, (2010). 


\section{Metodologia}

O diagnóstico dos fatores que dificultam o processo de ensino-aprendizagem em Química foi realizado na Escola Estadual Dias Cardoso Referência em Ensino Médio no $3{ }^{\circ} \mathrm{B}$, na cidade de Vitória de Santão Antão/PE, a escola faz parceria com PDVL (Programa Internacional Despertando Vocações para Licenciatura). Uma vez que, para a realização da intervenção houve aceitação e o acompanhamento por parte dos -dirigentes, professor, estudantes e responsáveis. Além disso, a pesquisa teve como natureza exploratória em que, levou-se em consideração a percepção e os motivos pela qual os alunos apresenta dificuldade nessa área com a participação de trinta alunos, realizando assim, um estudo de caso.

Para desenvolver essa pesquisa, foi aplicado um questionário que teve como questões direcionadas a entender a relação dos alunos com a Química, percebendo os principais motivos pela qual apresentam dificuldades ou não apresentam com essa área. Visto que, esses dados são importantes para a tomada de resolução coerente para minimizar ou melhorar essa situação.

É relevante que, a execução desta foi baseado na experiência do ciclo Kellyana, que é fundamentada na teoria de Construtos Pessoais de George Kelly. Esse ciclo é constituído por cinco etapas que se interealacionam: (i) Antecipação; (ii) Investimento; (iii) Encontro; (iv) Confirmação ou Desconfirmação; e (v) Revisão Construtiva. É necessário destacar a importância de se trabalhar voltado para o $3^{\circ}$ ano devido estarem em ano de vestibular.

Além do questionário houve a aplicação do teste de sondagem com conceitos mais corriqueiros utilizados em provas passadas do ENEM, na área de Ciências da Natureza, para a realização do mapeamento com os estudantes. Na aplicação do questionário e do teste de sondagem caracteriza-se a etapa de Antecipação do CEK. Sendo assim, os resultados destes revelam os motivos e os conteúdos de química por onde os alunos tem maior dificuldades.

Inicialmente foram mensurados os dados na qual apresenta os motivos do mal desenvolvimento dos alunos na área de Química. Mais adiante, houve a identificação do grau de facilidade de aprender química por parte dos estudantes. E por fim os conteúdos, na qual revelam maior dificuldade.

As intervenções com as aulas abrangem as atividades de Resolução de Problemas com base nas provas dos anos anteriores do ENEM na área de Ciências da Natureza. Nesse momento temos as etapas de Investimento e Encontro do CEK, onde foram ministradas aulas expositivas dialogadas com uso do quadro branco/pilotos e/ou por meio de slides sobre conteúdos que os estudantes apontaram com maior dificuldade segundo a avaliação diagnostica. A princípio, 
através das Resoluções de Problemas houve momentaneamente a contextualização com o diaa-dia dos estudantes, o levantamento de hipóteses, a estimulação do raciocínio promovendo assim uma aprendizagem de caráter significativo com o intuito de fortalecer o intelecto dos mesmos,

Para o Ensino de Química, Santos e Schnetzler (1996) propõem o desenvolvimento de atitudes e valores de participação social do indivíduo a partir do uso racional do conhecimento químico. Diante disso, os alunos podem argumentar de maneira crítica e reflexiva sobre o envolvimento entre o desenvolvimento científico, tecnológico, social e o ambiente onde eles se inserem com base na apropriação do conhecimento químico.

Após o encontro, a antepenúltima etapa do CEK que é a Confirmação ou Desconfirmação, os estudantes podem confirmar ou desconfirmar as suas hipóteses iniciais e verificar se os seus conhecimentos prévios levantados na etapa da Antecipação condizem com a vivência na prática. Verificam também se os estudos realizados durante as etapas de Investimento e Encontro os levaram à compreensão do que foi proposto. Finalizando o ciclo Kellyano com a Revisão Construtiva por onde os estudantes são motivados a cada finalização das aulas a refletirem sobre a aula. Em suma, questiona-se a percepção que os alunos atribuem a disciplina e o conteúdo ministrado antes e depois da disciplina, motivando-os a realizarem vestibulares para darem continuidade a esse processo de estudos e aprendizagem contribuindo para uma melhor educação.

\section{Resultados e Discussão}

Através dos resultados analisados, foi possível perceber que a maioria dos alunos afirmaram que a sua grande dificuldade em aprender os conteúdos de Química, são os cálculos. Os resultados revelam que 54,80\% dos estudantes tem dificuldades em cálculos; $48,40 \%$ apresentam dificuldade em compreender as aulas; 45,10\% têm dificuldades em decorar fórmulas; $25,80 \%$ não compreende bem as questões da prova e apenas 6,60\% não entende bem as aulas experimentais. Assim pode-se atentar que as aulas experimentais apreciam um bom desenvolvimento aos estudantes no quesito de uma boa compreensão nessa área, facilitando assim o processo de ensino-aprendizagem. 


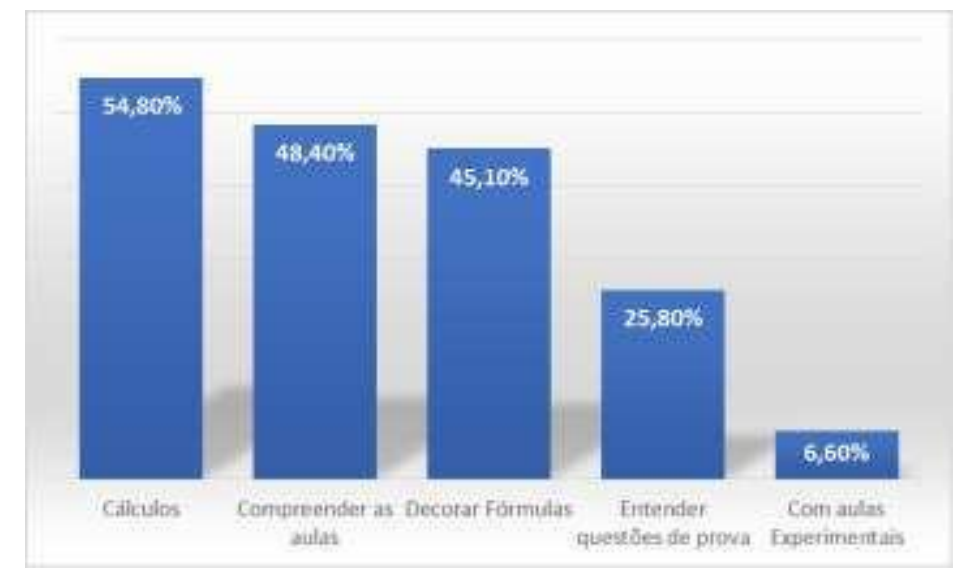

Cabe ressaltar, que esse resultado mostra bem a grande necessidade por parte dos docentes na busca de uma solução para tal problema. Diante disso, pode-se perceber que a solução não é tão simples. Além do mais, esses resultados deixam bem claro que os sujeitos possuem dificuldades em cálculos e consequentemente terão dificuldades na aprendizagem de determinados conceitos, por exemplo, estequiometria, pois a Química é uma ciência que explica os fenômenos naturais através das observações e utiliza esses como ferramenta operações matemáticas. Segundo Santos et al. (2013) essa dificuldade pode ser entendida como Discalculia, onde é observado um transtorno na realização de atividades envolvendo operações matemáticas sem apresentar distúrbios mentais ou motoras na realização do cálculo em uma idade cronológica específica. Esse transtorno pode ser de origem social, sem ser necessariamente de causas genéticas ou familiares (SANTOS et al., 2013).

Além disso outra questão foi indagada aos alunos, sendo assim os resultados comprovam que devido a carência no domínio de cálculos, consequentemente os alunos terão dificuldades em aprender. Nesse sentido, $58 \%$ dos alunos afirmaram que tem dificuldade em aprender os conteúdos de Química; 23\% admitem que não aprende; por fim apenas 19\% confirmam que aprende com facilidade. 
Gráfico 2. Grau de dificuldade de alunos em aprender os conteúdos.

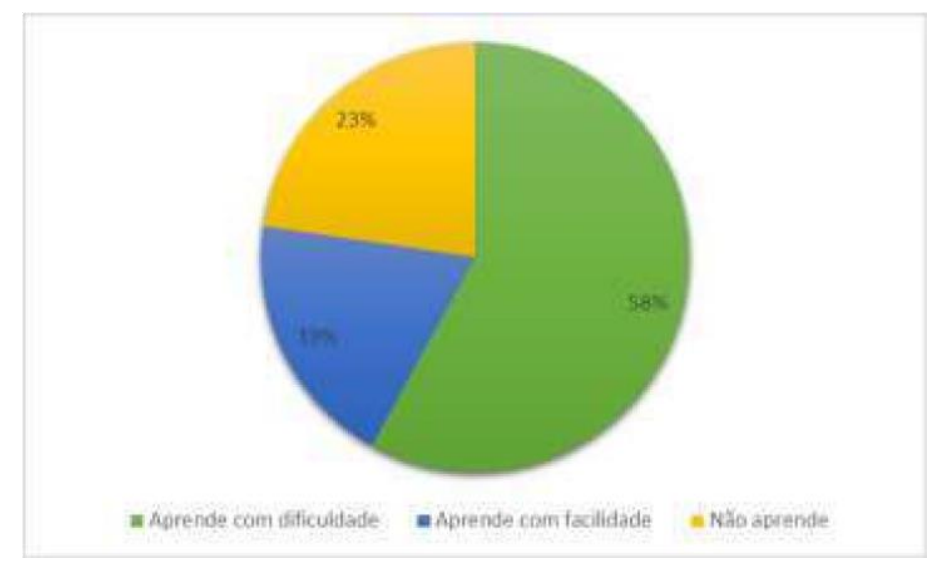

Outros dados que mencionados relevam outra premência para a finalidade dessa pesquisa, são os conteúdos em que os alunos apresentam dificuldades no processo de aprendizagem. Em particular foram sugeridos 21 assuntos de Química. Sendo assim; 70,1\% afirmaram que tem mais dificuldades em cálculos estequiométricos.

Gráfico,3. Dificuldades dos alunos do $3^{\circ}$ ano EREM- Dias Cardoso acerca dos conteúdos de Química.

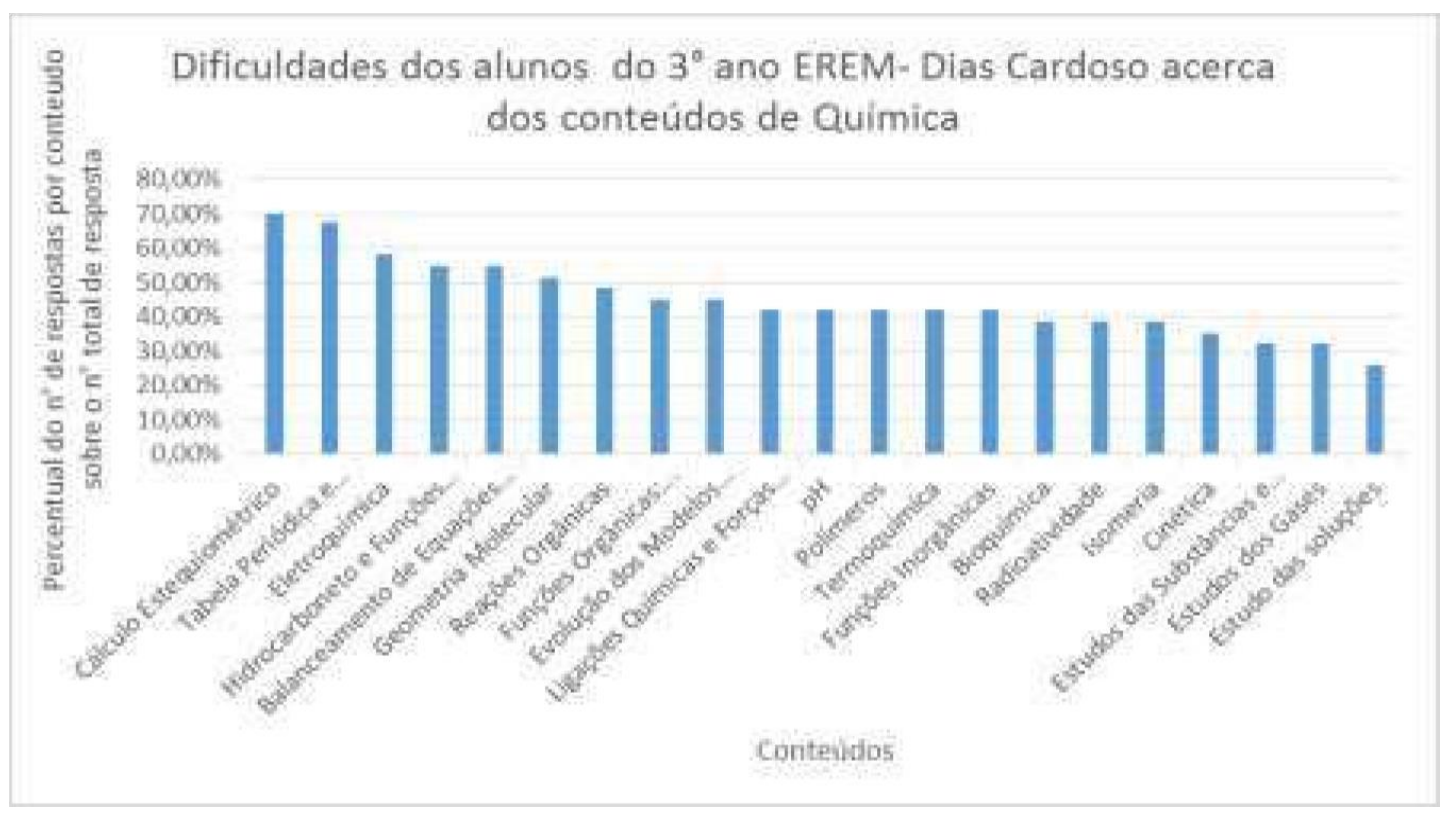

Analisando os gráficos pode-se perceber que os resultados se relacionam coerentemente, pois evidenciam que o maior impasse que dificultam a aprendizagem dos alunos são os cálculos, pois o conteúdo de cálculo estequiométrico foi o maior apontado.

Outra questão foi lecionada aos estudantes com o objetivo de saber a sua afinidade com a disciplina. Os resultados mostram que $58 \%$ dos estudantes gostam pouco da disciplina; 32,2\% 
afirmaram que gosta muito de Química; e 9,8\% não gostam.

Gráfico 4. Número de alunos que gostam, gostam pouco ou não gostam de Química.

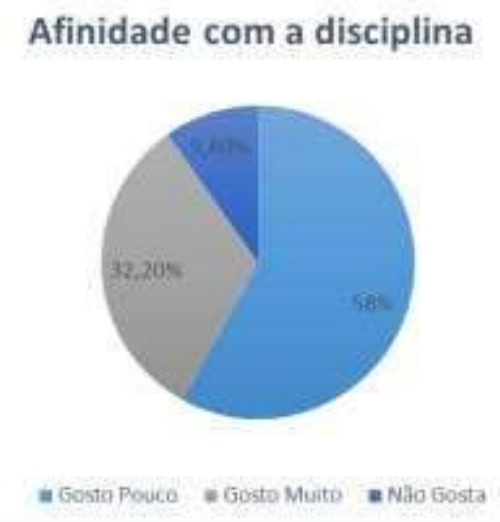

Os resultados mostram que, mesmo com dificuldade nessa área, existem uma quantidade relativamente grande de estudantes que gostam ou gostam pouco da disciplina.

Gráfico 5. Número de acertos e erros por questões do Teste de Sondagem. O gráfico 5 apresenta as 8 (oito) questões do teste mais há questões deixadas em branco.

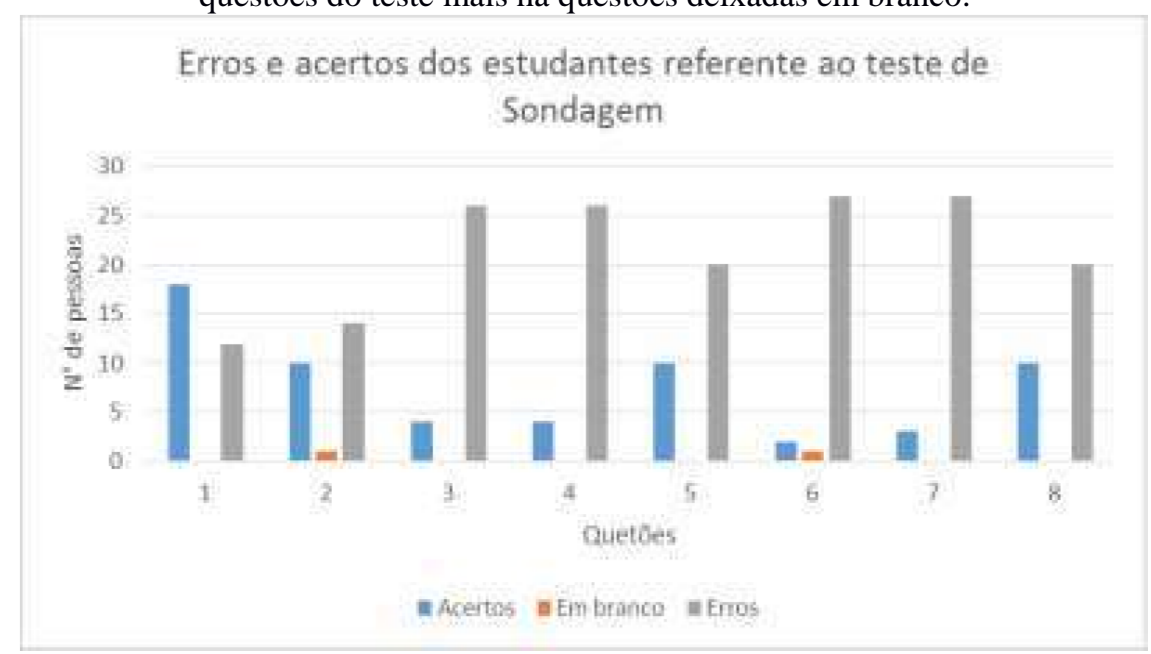

O teste de sondagem deixa claro que alunos apresentam muitas dificuldades nas resoluções de questões restrita ao ENEM. Contudo, os alunos mostraram um desempenho bom na primeira questão que envolve os assuntos de Funções Orgânicas em que, houve 18 acertos e 12 erros. Isso se deve pelo fato de ser um assunto abordado mais recentemente em sala de aula, pois no $3^{\circ}$ ano do ensino médio os conteúdos de Química Orgânica são ensinados. 


\section{Conclusões}

Em suma, diante dos questionários e o teste de sondagem está explícito que há vários adventos que contribui negativamente para aprendizagem dos conteúdos de Química, sendo os cálculos o maior impasse nesse processo, que exigem basicamente o raciocínio, as operações básicas da matemática, e atenção. Sendo assim é relevante que existem alternativas para minimizar essa situação, em que resoluções de problemas contextualizado restrito ao Exame Nacional do Ensino Médio (ENEM) possibilita um bom rendimento nessa área, pois estimulam os estudantes a raciocinarem, pensarem e serem mais ativos nesse processo, além de contribuir posteriormente aos processos seletivos. É de fato que existe a necessidade por parte dos docentes na busca de novas alternativas, pois os alunos não podem ficar nessa mesmice, nesse mundo inusitado em pensar que Química é algo difícil de aprender.

\section{Referências}

ANASTASIOU, L. G. C; ALVES, L. P. Processos de ensinagem na universidade: pressupostos para as estratégias de trabalho em aula. 10. ed. Joinville: UNIVILLE, 2012. 145p.

BRASIL. Ministério da Educação. Orientações Nacionais para o Ensino Médio. Ciências Matemática e Ciências da Natureza e suas Tecnologia. Brasília: Ministério da Educação (secretária da educação média e suas tecnologias), v.2, 2006.

FREIRE, Paulo. Pedagogia da autonomia: Saberes necessários à prática educativa. 37. ed. São Paulo: Paz e Terra, 2008

GASPARIN, João Luiz. Uma Didática para a Pedagogia Histórico-Crítica. $2^{a}$ ed. Campinas, SP: Autores Associados, 2003.

LACERDA, C. C., AMPOS, A. F., e MARCELINO-JR, C. A. C. (2012). Abordagem dos conceitos mistura, substância simples, substância composta e elemento químico numa perspectiva de ensino por situação-problema. Química Nova na Escola, 34(2), 75-82.

LIMA, J. O. G. Do período colonial aos nossos dias: uma breve história do Ensino de Química no Brasil. Espaço Acadêmico, Paraná, v. 12, n. 140, p. 71-79, 2013.

MIRANDA, D. G. P; COSTA, N. S. Professor de Química: Formação, competências/ habilidades e posturas. 2007

MEC (1998) Parâmetros curriculares nacionais: terceiro e quarto ciclos: apresentação dos temas transversais - 1998. Secretaria de Educação Fundamental, Ministério da Educação e do Desporto, Brasília, DF.

PARANÁ. Secretaria de Estado da Educação. Currículo básico para a escola pública do Paraná. Curitiba: SEED/DEPG, 1990. 
POLYA, G. Sobre a resolução de problemas de matemática na high school. In: KRULIK, S. E REYS, R. E. (org). A resolução de problemas na matemática escolar. São Paulo, Atual Editora, 1997. p. 1-3.

SANTOS, W. L. P., e SCHNETZLER, R. P. (2010). Educação em Química: Compromisso com a Cidadania. Ijuí: Unijuí.

SILVA, S. G. As principais dificuldades na aprendizagem de química na visão dos alunos do ensino médio. IX Congic, p. 1612-1616, julho 2013.

SANTOS, J. M. et al. As queixas escolares no Ensino de Ciências segundo professores da Rede Pública da Micro-Região de Floriano/PI. IN: FÓRUM INTERNACIONAL DE PEDAGOGIA.Vol. 1, 2013.

SANTOS, W. L.; SCHNETZLER, R. P. Função social: o que significa ensino de química para formar o cidadão? Química Nova na Escola, São Paulo, n. 4, p. 28-34, 1996.

VIANA, K. S. L. Avaliação da Experiência: uma perspectiva de Avaliação para o ensino das Ciências da Natureza. 2014. 202f. Tese (Ensino de Ciências - Física e Química) Universidade Federal Rural de Pernambuco - UFRPE, Recife, 2014

ZUCHI, Ivanete. A importância da linguagem no ensino de matemática. Educação Matemática em Revista, Recife, n. 16, p. 49 - 55, 2004 